\title{
PROTECTING LHC COMPONENTS AGAINST RADIATION RESULTING FROM COLLIDING BEAM INTERACTIONS*
}

\author{
N. V. Mokhov ${ }^{\dagger}$, I. L. Rakhno, FNAL, Batavia, IL 60510, USA
}

\section{Abstract}

Beam-induced energy deposition in the LHC high luminosity interaction region (IR) components due to both pp collisions and beam loss in the IR vicinity is a significant challenge for the design of the high luminosity insertions. It was shown in our previous studies that a set of collimators in the machine and absorbers within the lowbeta quadrupoles would reduce both the peak power density and total heat load to tolerable levels with a reasonable safety margin. In this paper the results of further optimization and comprehensive MARS calculations are briefly described for the updated IP1 and IP5 layouts and a baseline pp-collision source term. Power density, power dissipation, accumulated dose and residual dose rates are studied in the components of the inner triplets including their TAS absorbers, the TAN neutral beam absorbers, separation dipoles, and quadrupoles of the outer triplets and possible collimators there. It is shown that the optimized absorbers and collimators provide adequate protection of all the critical components.

\section{MARS MODELING IN IP1 AND IP5}

The Large Hadron Collider (LHC) [1] under construction at CERN, will produce pp collisions at $\sqrt{s}=14 \mathrm{TeV}$ and $L=10^{34} \mathrm{~cm}^{-2} \mathrm{~s}^{-1}$. The interaction rate of $8 \times 10^{8} \mathrm{~s}^{-1}$ represents a power of almost $900 \mathrm{~W}$ per beam, the large majority of which is directed towards the low- $\beta$ insertions. Previous studies [2,3] have identified this as a serious problem and proposed the ways to mitigate it. Below selected results of extensive studies of the IP1 and IP5 high luminosity insertions, performed for the latest lattice (version 6.2) with the newest version MARS 14 of the MARS code [4], are presented. All essential components situated in the tunnel of the IP1(R) and IP5(R) regions of $215 \mathrm{~m}$ long (up to the Q5 quadrupole) are implemented into the MARS 14 model with a detailed description of their geometry, materials and magnetic field distribution (Fig. 1). Horizontal crossing is modeled in the IP5 with correspondingly oriented beam pipes, while it is modeled vertically in the IP1. Near beam details of the ATLAS and CMS detectors are put in the model for the IP1 and IP5, respectively. Consideration is limited to luminosity-driven energy deposition effects in the inner and outer triplets. Impact of the circulating and misbehaved beam on the machine and detector components is considered elsewhere [5, 6].

\footnotetext{
* Work supported by the Universities Research Association, Inc., under contract DE-AC02-76CH03000 with the U. S. Department of Energy.

†mokhov@fnal.gov
}

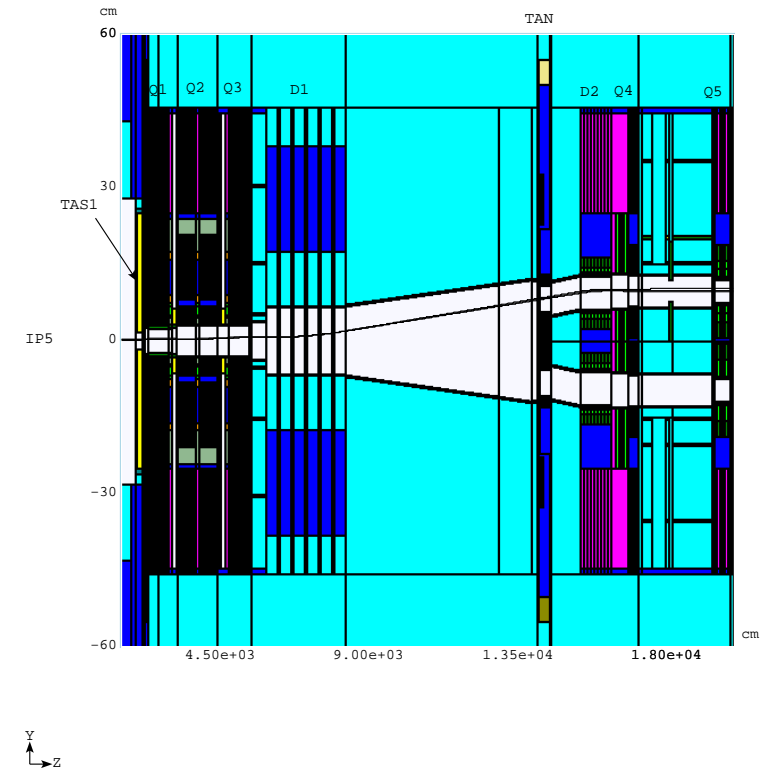

Figure 1: MARS model of the IP5 region. Proton tracks are shown in one ring.

\section{INNER TRIPLET}

The following protection system has been designed as a result of these studies: the TAS1 copper absorber (1.8-m long, $1.7 \mathrm{~cm}$ inner and $25 \mathrm{~cm}$ outer radii) at $19.45 \mathrm{~m}$ from the interaction point (IP), a stainless steel (SS) absorber $(23.5<\mathrm{r}<33.3 \mathrm{~mm})$ inside the $35-\mathrm{mm}$ radius $\mathrm{Q} 1$ aperture, a tapered SS liner in the MCBX, a TAS2 SS-copper absorber (1.1-m long, $25<\mathrm{r}<60 \mathrm{~mm}$ ) at $30.45 \mathrm{~m}$ from the IP in front of the Q2a quad, a TAS3 SS-copper absorber (1.2-m long, $33.3<\mathrm{r}<60 \mathrm{~mm}$ ) at $45.05 \mathrm{~m}$ from the IP in front of the Q3 quad, and a thicker beam pipe in the Q2a through Q3 region. Alternating magnetic field in the quads affects drastically the distribution of energy deposition $\epsilon$ in the inner triplet: $\epsilon$ peaks in horizontal and vertical planes and reaches maxima at a downstream or/and upstream end of the quads. There is a strong gradient in radial $\epsilon$-behavior. Fig. 2 shows a longitudinal distribution of an azimuthal peak in the first layers of the superconducting (SC) coils in the IP1(R) and IP5(R) inner triplets. These results are applicable to the other sides of the IRs, inverting the IP1(R)-IP5(L) and IP5(R)-IP1(L) pairs. The power density reaches its maximum $\epsilon_{\max }$, obviously, at $\beta_{\max }$ in the Q2b-Q3 region. This value is further increased in Q2b due to horizontal (IP5(R)) and (IP1(L)) crossings. With all of the above protective measures, one can keep $\epsilon_{\max }$ a factor of two to three-at the baseline luminosity—below the assumed quench limit of $1.2 \mathrm{~mW} / \mathrm{g}$. 


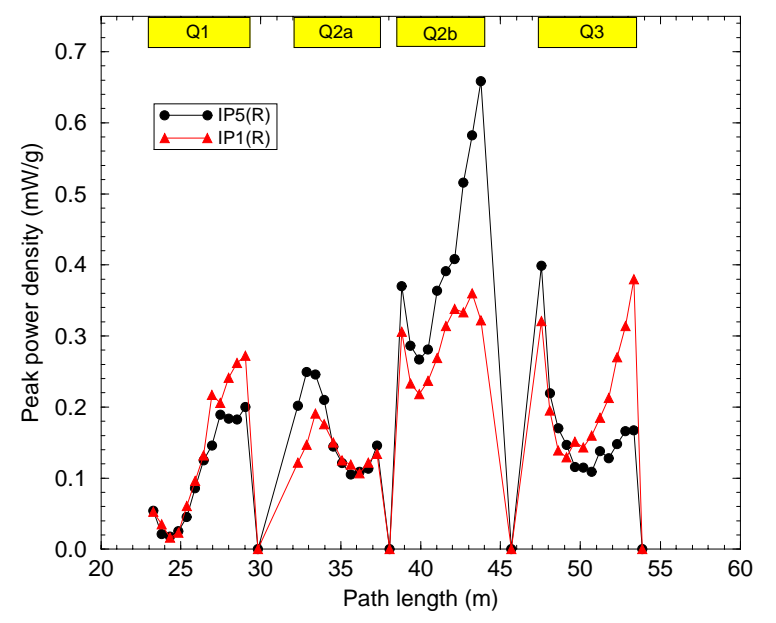

Figure 2: Peak power density in the first layer of the SC coils of the IP1 and IP5 inner triplet.

The absorbers do a very good job intercepting a signifi cant fraction of the energy escaping the colliding detectors. The TAS 1 alone absorbs $242 \pm 20 \mathrm{~W}$ on each side of the IP. The distribution of power dissipation in the IP5(R) inner triplet is shown in Fig. 3 separately for the coil regions, components inside the bore and for the remaining magnet mass. The Q1 inner absorber catches about $60 \%$ of power this region. The dose accumulated in the inner triplet components is quite high. For the corresponding baseline luminosity profile over an operational year, it can be estimated as $D(\mathrm{MGy} / \mathrm{yr})=7.8 \epsilon(\mathrm{mW} / \mathrm{g})$. The peak in the Q2b coilt can be as high as $4.7 \mathrm{MGy} / \mathrm{yr}$. Averaged over the coils at is about $100 \mathrm{kGy} / \mathrm{yr}$, dropping down to several $\mathrm{kGy} / \mathrm{yr}$ the slide bearings supporting the yoke. The later assumes that a 2-cm gap around the TAS1 core is filled before the collider run. Residual dose rates are quite significant in the near beam region - especially on the absorbers - being below $0.1 \mathrm{mSv} / \mathrm{hr}$ (30 days irradiation and 1 day cooling) on contact at the vessel (Fig. 4).

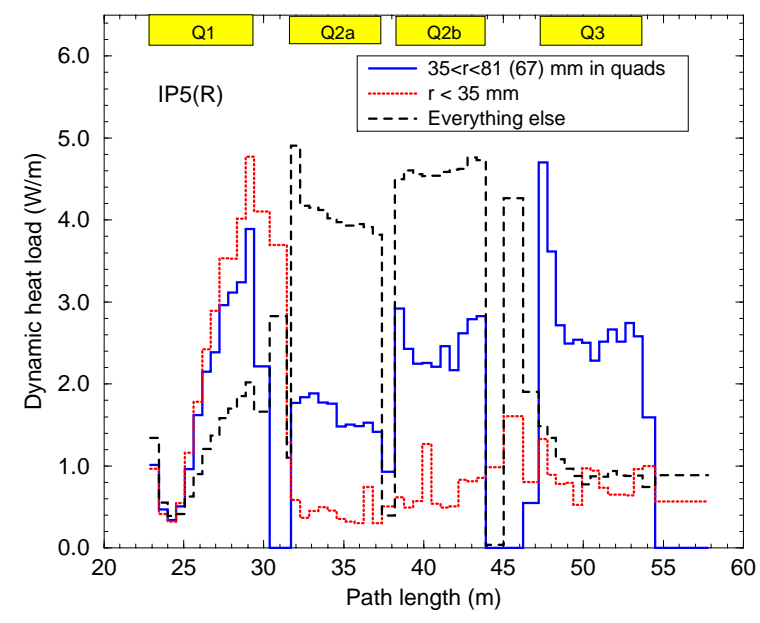

Figure 3: Power dissipation in the IP5 inner triplet.

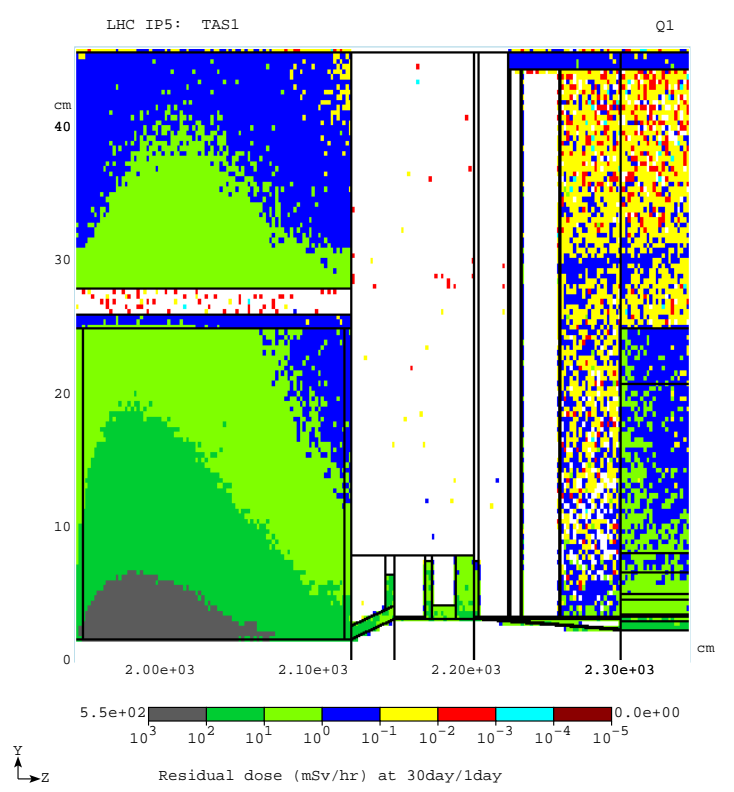

Figure 4: Residual dose (mSv/hr) for the IP5 TAS1-Q1.

\section{TAN, D2 AND OUTER TRIPLET}

A "neutral beam" absorber TAN at $140 \mathrm{~m}$ on each side of the IP, is designed to protect the separation dipoles D2 and the outer triplet quads [7]. Its parameters were optimized based on detailed MARS 14 calculations. An instrumented copper core $(21 \times 26 \times 350 \mathrm{~cm})$ with two $5 \mathrm{~cm}$ diameter beam holes is surrounded by massive steel shielding with a steel/marble albedo trap (Fig. 5). The power dissipated in the core is about $200 \mathrm{~W}$ and is brought primarily by energetic neutrals (45\% neutrons and $45 \%$ photons) generated at the IP and in the near beam components on a 140-m way from the IP. Residual dose rate on contact at the TAN outer surface of the steel shielding ( $y=+55 \mathrm{~cm}$ in Fig. 5) is shown in Fig. 6 for irradiation from 1 day to 20 years continuosly as a function of cooling time. In realistic operation, the dose is below $0.1 \mathrm{mSv} / \mathrm{hr}$ about a day after shutdown.

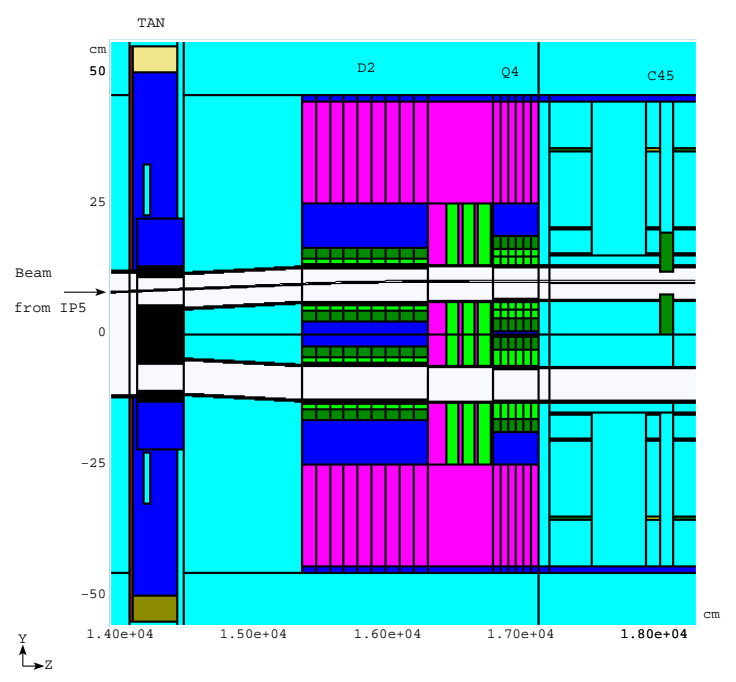

Figure 5: MARS model of the IP5 TAN and beginning of the outer triplet. Proton beam from the IP is shown. 


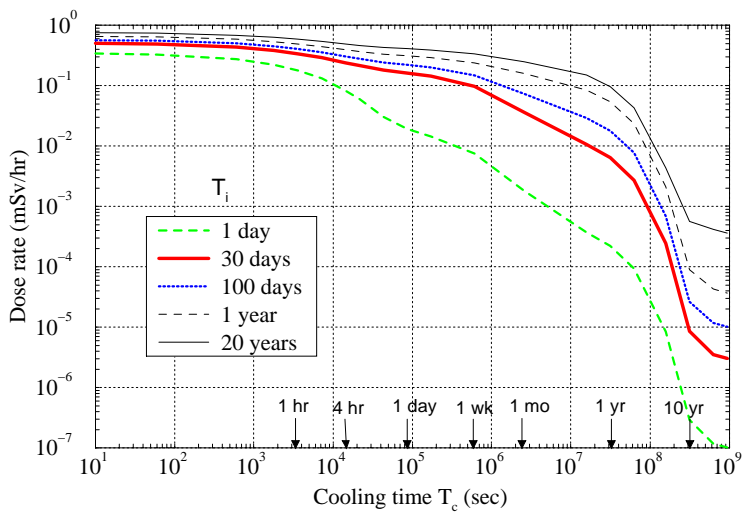

Figure 6: Residual dose (mSv/hr) averaged over the IP5 TAN shielding surface $(\mathrm{y}=+55 \mathrm{~cm})$ vs cooling time.

The TAN protects nicely the D2 dipole (Fig. 7) and Q4 quadrupole (Fig. 8), with the peak $\epsilon_{\max }$ in the SC coilswhich occurs again in a tiny azimuthal bin in the horizontal plane of the inner coil-almost a factor of hundred below the tolerable limit, with less than $1.75 \mathrm{~W}$ and $0.4 \mathrm{~W}$ of power dissipated in D2 and Q4, respectively. At the same time, calculations have shown that the peak power density in the Q5 SC coils was rather close to the allowable limit of $1.2 \mathrm{~mW} / \mathrm{g}$. It was found that an additional steel collimator C45 $(19.4 \times 19.4 \times 100 \mathrm{~cm})$, situated between Q4 and Q5 at $180.5 \mathrm{~m}$ from the IP and with a $21.3 \mathrm{~mm}$ aperture for the outgoing beam (see Fig. 5), solves this problem. Fig. 9 shows that both the peak power density in the SC coils and power dissipation in the Q5 quadrupole calculated with such a collimator are similar to those in D2 and Q4.

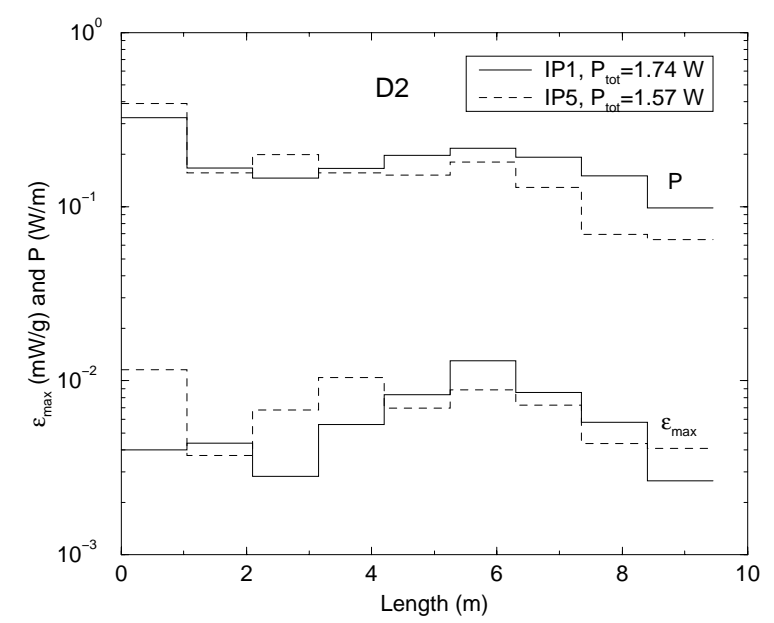

Figure 7: Peak power density $\epsilon_{\max }$ and dynamic heat load $P$ vs length in the IP1 and IP5 D2 separation magnet.

\section{REFERENCES}

[1] The Large Hadron Collider Conceptual Design, CERN/AC/95-05(LHC), 1995, P. Lefèvre and T. Pettersson, editors.

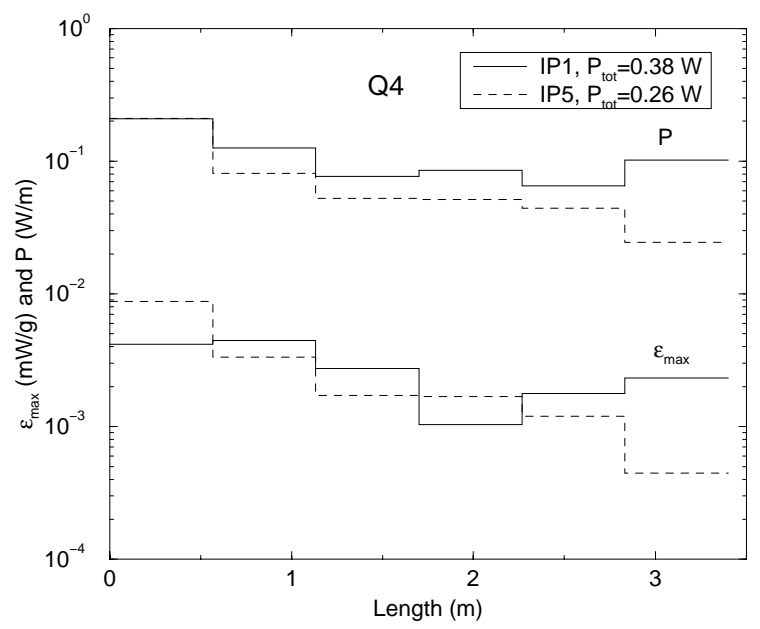

Figure 8: Peak power density $\epsilon_{\max }$ and dynamic heat load $P$ vs length in the IP1 and IP5 Q4 outer triplet quadrupole.

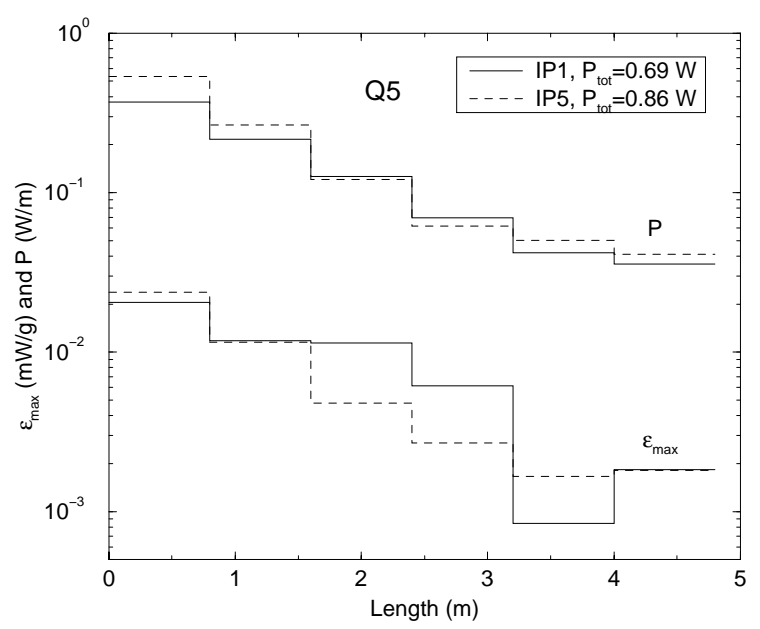

Figure 9: Peak power density $\epsilon_{\max }$ and dynamic heat load $P$ vs length in the IP1 and IP5 Q5 outer triplet quadrupole.

[2] N.V. Mokhov, "Accelerator-Experiment Interface at Hadron Colliders: Energy Deposition in the IR Components and Machine Related Background to Detectors", Fermilab-Pub94/085 (1994).

[3] N.V. Mokhov, J.B. Strait, Proc. of the 1997 Part. Accel. Conf., Vancouver, B.C., Canada, May 1997, p. 124.

[4] N. V. Mokhov, "The MARS Code System User's Guide", Fermilab-FN-628 (1995); N. V. Mokhov and O. E. Krivosheev, "MARS Code Status", Fermilab-Conf00/181 (2000). http://www-ap.fnal.gov/MARS/.

[5] A.I. Drozhdin, M. Huhtinen, N.V. Mokhov, Nucl. Instr. and Methods, A381, 531 (1996).

[6] A.I. Drozhdin, N.V. Mokhov, M. Huhtinen, Proc. of the 1999 Part. Accel. Conf., New York, 1999, p. 1231.

[7] E.H.Hoyer, W.C. Turner, N.V. Mokhov, Proc. of the 6th European Part. Accel. Conf., Stockholm, 1998, p. 368. 